\title{
No serological indications that systemic lupus erythematosus is linked with exposure to human parvovirus B19
}

\author{
Anders Bengtsson, Anders Widell, Sölve Elmståhl, Gunnar Sturfelt
}

\begin{abstract}
Objectives-Infectious agents like parvovirus have been implicated as exogenous factors that could trigger onset of systemic lupus erythematosus (SLE). A number of case reports describing a SLE-like presentation of acute human parvovirus B19 infection have been published, but no systematic investigation of the actual seroprevalence in epidemiologically defined SLE populations has previously been reported.

Methods-Sera from 99 SLE patients from a defined area in Southern Sweden, representing $88 \%$ of all new SLE cases 1981-1995 within the Lund-Orup Health Care district with 175000 adult inhabitants (> 15 years of age), and sera from 99 age and sex matched healthy controls were investigated for the presence of IgG parvovirus antibodies. Two different commercially available EIA kits were used; one using $E$ coli synthesised parvovirus VP1/VP2 antigen, and one using baculovirus derived parvovirus VP2 antigen.

Results-The EIA using baculovirus derived antigen was more sensitive and surprisingly the controls were more often positive than the SLE patients were $\mathbf{7 9 \%}$ versus $\left.65 \%, \chi^{2} p=0.027\right)$. No difference between the groups was seen with the EIA using $E$ coli derived antigen $(46 \%$ versus $49 \%$ ). Titration experiments indicated that the discordance between the two tests was a matter of sensitivity rather than specificity.
\end{abstract}

Department of Rheumatology, University Hospital, Lund, Sweden A Bengtsson G Sturfelt

Department of Clinical Microbiology,

University Hospital, Malmö, Sweden

A Widell

Department of Community Medicine, University Hospital, Malmö

S Elmståhl

Correspondence to: Dr A Bengtsson, Department of Rheumatology, Lund University Hospital, S-221 85 Lund, Sweden

Accepted for publication 20 August 1999 human parvovirus 19 infection being more prevalent among SLE patients. On the contrary, in one of the parvovirus EIAs the controls were more often positive than the SLE patients were.

(Ann Rheum Dis 2000;59:64-66)

Several infectious agents of viral origin have been suggested to trigger the onset of systemic lupus erythematosus (SLE). ${ }^{1-3}$ One of them is human parvovirus B19, a small single stranded DNA virus with tropism for erythroid precursor cells. It is the causative agent for erythema infectiosum (fifth disease), and disorders such as transient aplastic crisis in patients with haemolytic anaemia, stillbirth or hydrops fetalis when occurring during pregnancy, bone marrow suppression in the immunocompromised host, systemic vasculitis and both acute and chronic arthropathy. ${ }^{4}$ A large part of the population becomes exposed to parvovirus B19 during lifetime with seroprevalence increasing with age. ${ }^{5-7}$ Several case reports describe patients with an acute parvovirus B19 infection and SLE-like symptoms like rash, non-erosive arthritis, fever, anaemia, leucopenia, thrombocytopenia and positive ANA reviewed by Nesher et al. ${ }^{8}$ Furthermore, patients with acute parvovirus B19 infection may have antiphospholipid antibodies and also anti-dsDNA antibodies. ${ }^{9}{ }^{10}$ Despite the numerous case reports, no systematic investigation of the seroprevalence for parvovirus B19 in a SLE population has previously been performed to our knowledge. In this study, sera from 99 SLE patients from a defined area in southern Sweden, and sera from 99 age and sex matched healthy controls were investigated for the presence of anti-parvovirus B19 IgG antibodies with two immunoassays.

\section{Methods}

PATIENTS

The study group consisted of all incident cases of SLE retrieved 1981-1995 by validated methods ${ }^{11}$ within a defined area in southern Sweden. Thus, from the adult ( $>15$ years of age) population within the Lund-Orup Health Care district with 175000 adult inhabitants, 112 new cases were identified. Sera from 99 $(88 \%)$ of these 112 SLE patients were available for analysis. Sera from 99 sex and age matched healthy persons (mainly healthy hospital staff members and their friends and relatives) from the same recruitment area were used as controls. All sera were analysed for IgG antibodies to parvovirus B19.

Mean age of the SLE patients at the time of blood sampling was 49.6 years (median 48 , 17-89) and 12 of 99 were men. For controls mean age at the time of blood sampling was 48.6 years (median 47, 17-89), and 12 of the controls were men. Median number of American College of Rheumatology (ACR) classification criteria ${ }^{12}$ fulfilled was 5.0. The distribution of these criteria in the SLE group is shown in table 1.

PARVOVIRUS SEROLOGY

Determinations of parvovirus IgG antibodies were performed in parallel with two different enzymes linked immunosorbent assays (EIAs) based on recombinant prokaryote and eukaryote parvoviral antigens respectively:

The first test Parvovirus IgG Enzyme Immunoassay from Biotrin, Dublin, Ireland uses purified parvovirus VP2 antigen expressed 
Table 1 Distribution of ACR criteria among the 99 SLE patients

\begin{tabular}{llll}
\hline $\begin{array}{l}\text { ACR } \\
\text { number }\end{array}$ & & Number & $\%$ \\
\hline 1 & malar rash & 37 & 37 \\
2 & discoid rash & 30 & 30 \\
3 & photosensitivity & 59 & 60 \\
4 & oral ulcers & 16 & 16 \\
5 & arthtritis & 66 & 67 \\
6 & serositis & 48 & 48 \\
7 & renal disorder & 30 & 30 \\
8 & neurological disorder & 12 & 12 \\
9 & haematological disorder & 43 & 43 \\
10 & immunological disorder & 57 & 58 \\
11 & antinuclear antibodies & 99 & 100 \\
\hline
\end{tabular}

in baculovirus system, and was performed according to the manufacturer's instructions.

The second test Parvovirus IgG ELISA from Progen, Heidelberg, Germany is based on purified parvovirus B19 VP1 and VP2 antigens expressed in $E$ coli, also performed according to the manufacturer's instructions.

Anti-dsDNA IgG was measured with EIA (Euroimmun, Lübeck, Germany) and anticardiolipin IgG also with EIA (Euroimmun, Lübeck, Germany) according to the manufacturer's instructions.

\section{STATISTICS}

The $\chi^{2}$ method was used for group comparisons when comparing parvovirus B19 IgG positivity/negativity between SLE patients and controls, and between SLE patients with and without glomerulonephritis and/or CNS involvement. Spearman's rank correlation test was used for correlations between titres of parvovirus B19 IgG and titres of anti-dsDNA IgG and anti-cardiolipin IgG antibodies, and the number of ACR criteria fulfilled.

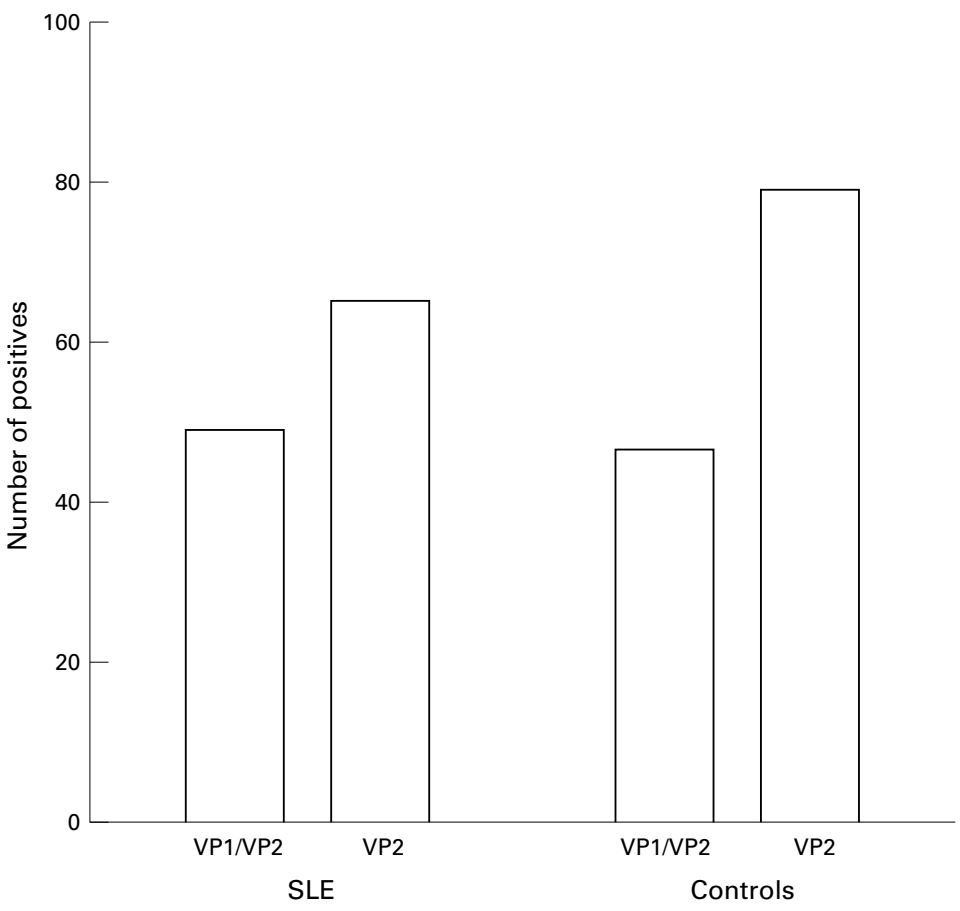

Figure 1 Number of positives in the two different parvovirus B19 EIAs. No difference was seen between the 99 SLE patients and the 99 controls with the IgG VP1/VP2 EIA (Progen), $49 \%$ of the SLE patients were positive compared with $46 \%$ of the controls. The VP2 EIA (Biotrin) was more sensitive and in this case the controls were significantly more often positive than the SLE patients ( $p=0.027$ ), $65 \%$ of the SLE patients were positive compared with $79 \%$ of the controls.

\section{Results}

The EIA based on baculovirus derived parvovirus B19 VP2 (Biotrin) was the most sensitive (see below) and with this assay the healthy controls were slightly more often positive than the SLE patients $(p=0.027)$. Seventy nine per cent of the controls were positive compared with $65 \%$ of the SLE patients (fig1).

No difference was seen between SLE patients and controls with the IgG $E$ coli derived VP1/VP2 EIA (Progen), 49\% of the SLE patients being positive compared with $46 \%$ of the controls (fig 1 ).

Thirty three per cent of the SLE patients were positive for anti-cardiolipin (aCL) antibodies and $26 \%$ were positive for anti-dsDNA antibodies. No correlations between titres of antibodies against B19 antigens and aCL antibodies or anti-dsDNA antibodies were observed.

To investigate if there was a relation between disease severity and parvovirus B19 reactivity, patients with glomerulonephritis and/or CNS involvement (ACR criteria 7 and 8) were compared with patients without these manifestations. With both assays patients with kidney and/or CNS involvement were more often parvovirus B19 negative (VP2 assay: $\mathrm{p}=0.037$, VP1/VP2 assay: $p=0.018)$. Mean age in these two groups of SLE patients were similar (48.4 versus 50.5), and a difference in age could thus not explain these results. However, no relation was observed between the number of ACR criteria and parvovirus B19 reactivity.

We found some discrepancies between the results with the two assays: positivity in the VP2 (Biotrin) assay/negativity in the VP1/VP2 (Progen) assay. Sera from 15 SLE patients positive in the Biotrin EIA but negative in the Progen EIA were therefore tested further. These 15 discordant sera together with seven concordant IgG positive sera were also assayed by the Biotrin IgG EIA at higher dilutions $(1 / 800,1 / 1600)$ than dilution $1 / 100$ recommended by the manufacturer (1/100). At serum dilution $1 / 800$ all seven concordant sera but only 3 of 15 discordant sera reacted above cut off in the Biotrin test. At serum dilution $1 / 1600$ all seven concordant sera were still positive, whereas only 1 of 15 discordant sera was positive, indicating that the titres in the discordant samples were lower. In addition, the same 15 discordant sera were retested by the Progen assay at a lower dilution $(1 / 50)$ than recommended by the manufacturer (1/100) together with five samples that were concordantly negative by both Biotrin and Progen. Of the 15 discordant samples seven turned positive at dilution $1 / 50$ whereas all five concordantly negative samples remained negative in the Progen assay.

Taken together, these findings strongly indicate that the discordance between the test results was attrributable to a higher test sensitivity of the Biotrin test to detect low titred samples and not attributable to lower specificity. 


\section{Discussion}

Patients with an acute parvovirus B19 infection may present with SLE-like symptoms ${ }^{8}$ and SLE associated autoantibodies. ${ }^{9}{ }^{10}$ This prompted us to investigate the prevalence of parvovirus B19 infection in an epidemiologically recruited cohort of SLE patients and a set of matched controls. The SLE cohort represented all new cases during 1981-1995 in a defined area in southern Sweden, and from a majority of these $(88 \%)$ serum samples were available for parvovirus B19 testing.

Anti-parvovirus B19 IgG seroprevalence was not increased versus controls. This is the key observation of our study. Instead the opposite finding was, somewhat unexpectantly, obtained by the most sensitive assay. Thus, in the VP2 EIA (Biotrin) $65 \%$ of the SLE patients were positive compared with $79 \%$ of the controls ( $p$ $=0.027)$. The antibody prevalence is of the same magnitude as in other studies from northern Europe..$^{5-7}$ The slightly lower prevalence rate of anti-parvovirus B19 VP2 IgG in the SLE group, could be a question of titres as we observed a difference in sensitivity between the two assays. Another possibility is influence of drug treatment, especially among patients with kidney and/or CNS involvement.

Human parvovirus B19 is more commonly, together with HIV, hepatitis B and C viruses, and Epstein-Barr virus, associated with the production of autoantibodies than other viruses. ${ }^{13}$ In the case reports of parvovirus B19 infected patients with SLE-like presentations, presence of ANA, anti-dsDNA-, aCL-, antiSS-A- and SS-B-antibodies have been described. It has also been observed that the specificity of the aCL antibodies found in parvovirus B19 infected patients is similar to the specificity of the aCL antibodies in SLE patients. ${ }^{9}$ In addition, the frequency of antidsDNA antibodies among patients with acute parvovirus B19 infection has been reported to be high. ${ }^{10}$ In our SLE cohort, the frequencies of anti-dsDNA and aCL antibodies were when determined at the same time point as antibodies to parvovirus B19 was measured $26 \%$ and $33 \%$ positivity, respectively. The frequencies were as to be expected in an unselected SLE population. $^{1415}$ No association was found between these autoantibodies and IgG antibodies to human parvovirus B19.

Our study does not exclude the possibility that parvovirus B19 might be a trigger of SLE disease in a minor subset of SLE patients. However, the main conclusion is that in the general population parvovirus B19 is unlikely to be of aetiological importance for SLE. Funding: grants were obtained from the Medical Faculty of the
University of Lund, Alfred Österlunds Foundation, Greta and University of Lund, Alfred Osterlunds Foundation, Greta and Johan Kock's Foundation, King Gustav V:s 80th Birthday
Fund, Medical Faculty of University of Lund, Prof Nanna Fund, Medical Faculty of University of Lund, Prof Nanna
Svartz' Fund, The Swedish National Association against Rheumatism

1 James JA, Kaufman KM, Farris AD, Taylor-Albert E, Lehman TJA, Harley JB. An increased prevalence of EpsteinBarr virus infection in young patients suggests a possible tiology for systemic lupus erythematosus. J Clin Invest 1997;100:3019-26.

2 Herrmann M, Hagenhofer M, Kalden JR. Retroviruses and systemic lupus erythematosus. Imm Review 1996;152: systemic $145-56$.

3 Rider JR, Ollier WE, Lock RJ, Brookes ST, Pamphilon DH. Human cytomegalovirus infection and systemic lupus erythematosus. Clin Exp Rheumatol 1997;15:405-9.

4 Török TJ. Parvovirus B19 and human disease. Adv Intern Med 1992;37:431-55

5 Cohen BJ, Buckley MM. The prevalence of antibody to human parvovirus B19 in England and Wales. J Med Microbiol 1988;25:151-3.

6 Eis-Hubinger AM, Oldenburg J, Brackmann HH, Matz B, Schneweis KE. The prevalence of antibody to parvovirus B19 in hemophiliacs and in the general population. Zentralbl Bakteriol 1996;284:232-40.

7 Skjoldebrand-Sparre L, Fridell E, Nyman M, Wahren B. A prospective study of antibodies against parvovirus B19 in pregnancy. Acta Obstet Gynecol Scand 1996;75:336-9.

8 Nesher G, Osborn TG, Moore TL. Parvovirus infection mimicking systemic lupus erythematosus. Semin Arthritis mimicking systemic lupus

9 Loizou S, Cazabon JK, Walport MJ, Tait D, So AK. Similarities of specificity and cofactor dependence in serum antiphospholipid antibodies from patients with human parvovirus B19 infection and from those with systemic lupus erythematosus. Arthritis Rheum 1997;40: 103-8.

10 Soloninka CA, Anderson MJ, Laskin CA. Anti-DNA and antilymphocyte antibodies during acute infection with human parvovirus B19. J Rheumatol 1989;16:777-81.

11 Jonsson H, Nived O, Sturfelt G. Outcome in systemic lupus erythematosus: a prospective study of patients from a defined population. Medicine (Baltimore) 1989;68:141-

12 Tan EM, Cohen AS, Fries JF, Masi AT, McShane DJ, Rothfield NF, et al. The 1982 revised criteria for the classification of systemic lupus erythematosus. Arthritis Rheum 1982;25:1271-7.

13 Hansen KE, Arnason J, Bridges AJ. Autoantibodies and common viral illnesses. Semin Arthritis Rheum 1998;27: common

14 Sturfelt G, Nived O, Norberg R, Thorstensson R, Krook K. Anticardiolipin antibodies in patients with systemic lupus erythematosus. Arthritis Rheum 1987;30:382-8.

15 Eaton RB, Schnneider G, Schur PH. Enzyme immunoassay for antibodies to native DNA. Specificity and quality of antibodies. Arthritis Rheum 1983;26:52-62. 